\title{
Brain-Computer Interface Technology: A Review of the First International Meeting
}

Jonathan R. Wolpaw (Guest Editor), Niels Birbaumer, William J. Heetderks, Dennis J. McFarland, P. Hunter Peckham, Gerwin Schalk, Emanuel Donchin, Louis A. Quatrano, Charles J. Robinson, and Theresa M. Vaughan (Guest Editor)

\begin{abstract}
Over the past decade, many laboratories have begun to explore brain-computer interface (BCI) technology as a radically new communication option for those with neuromuscular impairments that prevent them from using conventional augmentative communication methods. BCI's provide these users with communication channels that do not depend on peripheral nerves and muscles. This article summarizes the first international meeting devoted to BCI research and development. Current BCI's use electroencephalographic (EEG) activity recorded at the scalp or single-unit activity recorded from within cortex to control cursor movement, select letters or icons, or operate a neuroprosthesis. The central element in each BCI is a translation algorithm that converts electrophysiological input from the user into output that controls external devices. BCI operation depends on effective interaction between two adaptive controllers, the user who encodes his or her commands in the electrophysiological input provided to the BCI, and the BCI which recognizes the commands contained in the input and expresses them in device control. Current BCI's have maximum information transfer rates of 5-25 b/min. Achievement of greater speed and accuracy depends on improvements in signal processing, translation algorithms, and user training. These improvements depend on increased interdisciplinary cooperation between neuroscientists, engineers, computer programmers, psychologists, and rehabilitation specialists, and on adoption and widespread application of objective methods for evaluating alternative methods. The practical use of BCI technology depends on the development of appropriate applications, identification of appropriate user groups, and careful attention to the needs and desires of individual users. BCI research and development will also benefit from greater emphasis on peer-reviewed publications,
\end{abstract}

Manuscript received March 3, 2000; revised March 13, 2000. The National Center for Medical Rehabilitation Research of the National Institute of Child Health and Human Development of the NIH provided major funding for this workshop. The Eastern Paralyzed Veterans Association and the Whitaker Foundation supported graduate students and postdoctoral fellows.

J. R. Wolpaw, D. J. McFarland, G. Schalk, and T. M. Vaughan are with the Laboratory of Nervous System Disorders, Wadsworth Center, New York State Department of Health and State University of New York, Albany, NY 12201 USA.

N. Birbaumer is with the Institute of Medical Psychology and Behavioral Neurobiology, University of Tuebingen, Tuebingen, Germany.

W. J. Heetderks is with the Division of Fundamental Neurosciences, National Institute of Neurological Disorders and Stroke, National Institutes of Health, Bethesda, MD USA.

P. H. Peckham is with the Rehabilitation Engineering Center, Metro Health Center, Case Western Reserve University, Cleveland, OH 44109 USA.

E. Donchin is with the Beckman Institute, University of Illinois, Champaign, IL 61820 USA.

L. A. Quatrano is with the National Center for Medical Rehabilitation Research, National Institute of Child Health and Human Development, National Institutes of Health, Bethesda, MD USA.

C. J. Robinson is with the Center for Biomedical Engineering and Rehabilitation Science, Louisiana Tech University, Ruston, LA 71270 USA. He is aslo with Overton Brooks VA Medical Center, Shreveport, LA 71101 USA (e-mail: c.robinson@ieee.org).

Publisher Item Identifier S 1063-6528(00)04484-0. and from adoption of standard venues for presentations and discussion.

Index Terms-Brain-computer interface (BCI), electroencephalography (EEG), augmentative communication.

\section{INTRODUCTION}

Brain-computer interfaces (BCI's) give their users communication and control channels that do not depend on the brain's normal output channels of peripheral nerves and muscles. Current interest in BCI development comes mainly from the hope that this technology could be a valuable new augmentative communication option for those with severe motor disabilities-disabilities that prevent them from using conventional augmentative technologies, all of which require some voluntary muscle control. Over the past five years, the volume and pace of BCI research have grown rapidly. In 1995 there were no more than six active BCI research groups, now there are more than 20 . They are focusing on brain electrical activity, recorded from the scalp as electroencephalographic activity (EEG) or from within the brain as single-unit activity, as the basis for this new communication and control technology.

In recognition of this recent rapid development and its potential importance for those with motor disabilities, the National Center for Medical Rehabilitation Research of the National Institute of Child Health and Human Development of the National Institutes of Health sponsored a workshop on BCI technology. This workshop, also supported by the Eastern Paralyzed Veterans Association and the Whitaker Foundation and organized by the Wadsworth Center of the New York State Department of Health, took place in June of 1999 at the Rensselaerville Institute near Albany, New York. Fifty scientists and engineers participated. They represented 22 different research groups from the United States, Canada, Great Britain, Germany, Austria, and Italy. Their principal goals were: 1) to review the current state of BCI research; 2) to define the aims of basic and applied BCI research; 3) to identify and address the key technical issues; and 4) to consider development of standard research procedures and assessment methods.

On the first day, one person from each group gave a brief summary of his or her group's current work and future plans. The substance of these talks is presented in the peer-reviewed papers that follow this article. They range from descriptions of a variety of functioning EEG-based or single-unit based BCI's, to analyses of the correlations between EEG or single-unit activity and the brain's conventional motor outputs, to investigations of 
issues important for BCI applications, to BCI software development. Together they constitute a comprehensive review of the present state of BCI research.

The following two days were devoted to six discussion sessions, each led by a panel of five to seven people; and each addressing a set of questions focused on a single important aspect of BCI research and development. Evenings were occupied with demonstrations of BCI technology and by poster presentations. The discussion sessions were designed to cover the full range of crucial issues, from the essential features of any BCI, to the brain activity it uses, to the algorithms that translate that activity into control signals, to user-system interactions, to research methods and standards, to practical applications in rehabilitation settings. The sections that follow, written by the panel chairmen, summarize the contents and conclusions of these discussions. Taken together, these summaries touch on each key issue at least once and often more than once and in different ways.

\section{SESSION 1: DEFINITION AND ESSENTIAL FEATURES OF A BRAIN-COMPUTER INTERFACE (BCI)}

Since the EEG was first described by Hans Berger in 1929 [1], people have speculated that it might be used for communication and control, that it might allow the brain to act on the environment without the normal intermediaries of peripheral nerves and muscles. In the 1970's, several scientists developed simple communication systems that were driven by electrical activity recorded from the head. Early in that decade, the Advanced Research Projects Agency (ARPA, which also sponsored the initial development of the internet) of the U.S. Department of Defense became interested in technologies that provided a more immersed and intimate interaction between humans and computers and included so-called "bionic" applications. A program proposed and directed by Dr. George Lawrence focused initially on autoregulation and cognitive biofeedback. It sought to develop biofeedback techniques that would improve human performance, especially the performance of military personnel engaged in tasks that had high mental loads. The research produced some valuable insights on biofeedback, but made minimal progress toward its stated goals. A new direction, under the more general label of "biocybernetics," was then defined and became the main source of support for bionics research in the ensuing years. One of the directives of the biocybernetics program was to evaluate the possibility that biological signals, analyzed in real-time by computer, could assist in the control of vehicles, weaponry, or other systems. The most successful project in this area was that headed by Dr. Jacques Vidal, Director of the Brain-Computer Interface Laboratory at UCLA. Using computer-generated visual stimulation and sophisticated signal processing, the research showed that single-trial (i.e., not averaged) visual evoked potentials (VEP's) could provide a communication channel by which a human could control the movement of a cursor through a two-dimensional maze [2].

Vidal's studies and other less well-controlled early work brought out the importance of the distinction between control systems that use actual EEG activity and those that use EMG (electromyographic) activity from scalp or facial muscles.
Because scalp-recorded EMG activity can be much more prominent than EEG activity at the same locations, EMG-based communication can masquerade as EEG-based communication. To the extent that EMG-based communication is mistaken for EEG-based communication, it can hamper the latter's development. Careful spectral and topographical analysis may be needed to distinguish one from the other. The early work also served to bring out the fundamental distinction between EEG-based communication that depends on muscle control (e.g., visual evoked potentials that depend on where the eyes are directed), and EEG-based control that does not depend on muscle control.

These distinctions shaped the definition of the term BCI put forward in this session: "A brain-computer interface is a communication system that does not depend on the brain's normal output pathways of peripheral nerves and muscles." This definition also reflects the principal reason for recent interest in BCI development - the possibilities it offers for providing new augmentative communication technology to those who are paralyzed or have other severe movement deficits. All other augmentative communication technologies require some form of muscle control, and thus may not be useful for those with the most severe motor disabilities, such as late-stage amyotrophic lateral sclerosis, brainstem stroke, or severe cerebral palsy.

As a number of the presentations at this conference demonstrated, several different true BCI's, that is, communication systems that do not appear to depend on nerves and muscles, have been achieved (e.g., [3]-[9]). These systems use either EEG activity recorded from the scalp or the activity of individual cortical neurons recorded from implanted electrodes. While these are exciting developments, with considerable theoretical significance and practical promise, they are relatively low bandwidth devices, offering maximum information transfer rates of 5-25 bits/min at best. Furthermore, improvement is likely to be gradual, and to require continued careful and laborious investigation.

BCI development requires recognition that a "wire-tapping" analogy probably does not apply-that the goal is not simply to listen in on brain activity (via EEG, intracortical recording, or some other method) and thereby determine a person's intentions. A BCI is a new output channel for the brain, and, like the brain's normal output channels of peripheral nerves and muscles, is likely to engage the brain's adaptive capacities, which adjust output so as to optimize performance. Thus, BCI operation depends on the interaction of two adaptive controllers, the user's brain, which produces the activity measured by the BCI system, and the system itself, which translates that activity into specific commands. Successful BCI operation is essentially a new skill, a skill that consists not of proper muscle control but rather proper control of EEG (or single-unit) activity.

Like any communication and control system, a BCI has an input, an output, and a translation algorithm that converts the former to the latter. BCI input consists of a particular feature (or features) of brain activity and the methodology used to measure that feature. As the BCI and pre-BCI studies presented at this workshop illustrate, BCI's may use frequency-domain features (such as EEG $\mu$ or $\beta$ rhythms occurring in specific areas of cortex) [6]-[9] and [11]-[14], or time-domain features (such as 
slow cortical potentials, $\mathrm{P} 300$ potentials, or the action potentials of single cortical neurons) [3]-[5], [10], [12], [15]-[18]. The methodology includes the scalp electrode type and locations, the referencing method, the spatial and temporal filters, and other signal-processing methods used to detect and measure the features. The distinction between a feature as a reflection of a specific aspect of nervous system physiology and anatomy and a methodology as a technique for measuring the feature is more clear for some features (e.g., the firing rate of a single cortical neuron, which is presumably the same however it is measured) than for others (e.g., autoregressive parameters, which depend on the details of the analysis algorithm). Nevertheless, the distinction is important because attention to features as reflections of nervous system anatomy and physiology, rather than as merely products of particular analysis methods, helps guide improvements in BCI technology, and also encourages continued attention to the problem of artifacts such as EMG activity (which can, for example, affect autoregressive parameters).

Each BCI uses a particular algorithm to translate its input (e.g., its chosen EEG features) into output control signals. This algorithm might include linear or nonlinear equations, a neural network, or other methods, and might incorporate continual adaptation of important parameters to key aspects of the input provided by the user. BCI outputs can be cursor movement, letter or icon selection, or another form of device control, and provides the feedback that the user and the BCI can use to adapt so as to optimize communication.

In addition to its input, translation algorithm, and output, each BCI has other distinctive characteristics. These include its On/Off mechanism (e.g., EEG signals or conventional control); response time, speed and accuracy and their combination into information transfer rate; type and extent of user training required, appropriate user population; appropriate applications; and constraints imposed on concurrent conventional sensory input and motor output (e.g., the need for a stereotyped visual input, or the requirement that the user remain motionless).

Because BCI operation depends on the user encoding his or her wishes in the EEG (or single-unit) features that the system measures and translates into output control signals, progress depends on development of improved training methods. Future studies should evaluate the effects of the instructions given to users, and analyze the relationships between user reports of strategies employed and actual BCI performance. For example, some BCI protocols ask that the user employ very specific motor imagery (e.g., imagery of right or left hand movement) or other mental tasks to produce the EEG features the system uses as control signals (e.g., [7], [9]). Others may leave the choice of imagery, or the decision to use any imagery at all, up to the user (e.g., [3], [8]). Analysis of the similarities and differences between acquisition of BCI control and acquisition of conventional motor or nonmotor skills could lead to improvements in training methods. The impacts of subject motivation, fatigue, frustration, and other aspects of mental states also require exploration. Users' reports might help in assessing these factors. At the same time, the value of such reports is not clear. Users' reports of their strategies may not accurately reflect the processes of achieving and maintaining EEG control (e.g., [19]).

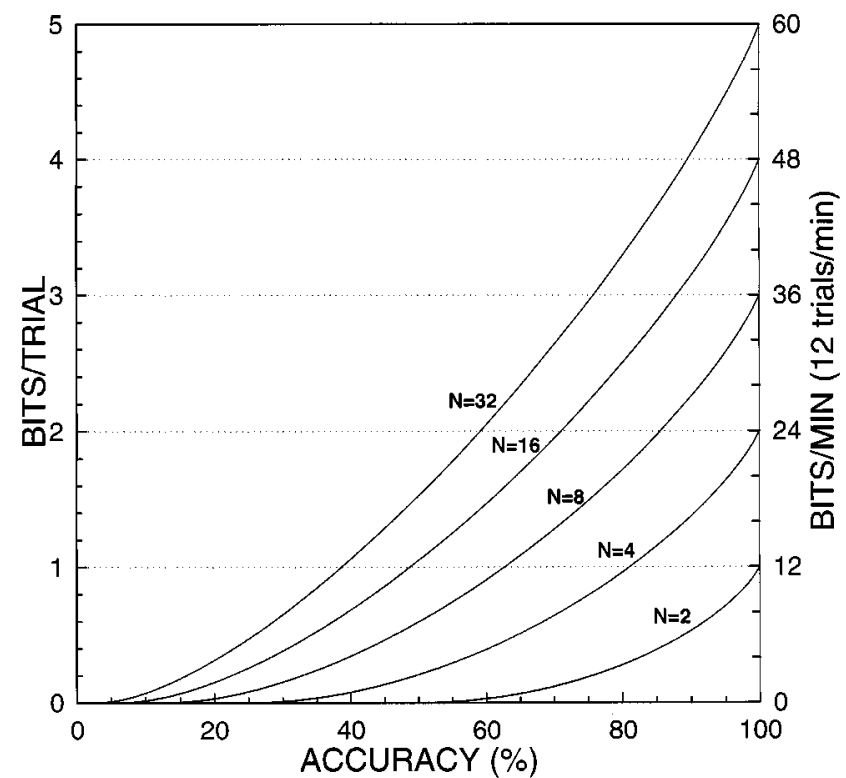

Fig. 1. Information transfer rate in bits/trial (i.e., bits/selection) and in bits/min (for 12 trials/min) when the number of possible selections (i.e., $\mathrm{N}$ ) is $2,4,8,16$, or 32. As derived from Pierce [21] (and originally from Shannon and Weaver [20]), if a trial has $N$ possible selections, if each selection has the same probability of being the one that the user desires, if the probability $(P)$ that the desired selection will actually be selected is always the same, and if each of the other (i.e., undesired) selections has the same probability of selection (i.e., $(1-P) /(N-1))$, then bit rate, or bits/trial $(B)$, is: $B=\log _{2} N+P \log _{2} P+(1-P) \log _{2}[(1-P) /(N-1)]$. For each $N$, bit rate is shown only for accuracy $\geq 100 / N$ (i.e., $\geq$ chance).

Because BCI's differ greatly in their inputs, translation algorithms, outputs, and other characteristics, they are often difficult to compare. While it is likely that different systems will prove most valuable for different applications, a standard performance measure would be useful as a general purpose benchmark for following BCI development. A standard measure of communication systems is bit rate, the amount of information communicated per unit time. Bit rate depends on both speed and accuracy [20], [21]. Fig. 1 illustrates the relationship between accuracy and information transfer rate for different numbers of choices (i.e., 2, 4, 8, 16, 32). Information transfer rate is shown both as bits/trial (i.e., bits/selection), and as bits/min when 12 selections are made each min [a selection rate similar to that of several current BCI's (e.g., [3], [7], [8])]. Thus, for example, the information transfer rate of a BCI that can select between two possible choices with $90 \%$ accuracy is twice that of a BCI that can select between them with $80 \%$ accuracy, and equal to that of a BCI that can select between four possible choices with 65\% accuracy. The enormous importance of accuracy, illustrated by the doubling in information transfer rate with improvement from $80 \%$ to $90 \%$ accuracy in a two-choice system, has not usually received appropriate recognition in BCI-related publications. While the effectiveness of each BCI system will depend in considerable part on the application to which it is applied, bit rate furnishes an objective measure for comparing different systems and for measuring improvements within systems.

The continuation and acceleration of BCI development and application does not depend solely on scientific and technical advances. It depends also on attention to important practical 
issues. At present, the pace of development is limited by the small number of people involved and the relatively modest funding available. Increased collaboration, fostered in part by meetings such as this BCI conference, and increased numbers of refereed publications in high-quality journals, should encourage more funding from public and private agencies worldwide. On the other hand, naive and overly enthusiastic media attention is likely to be detrimental in the long run. Major funding increases, particularly for development of specific applications, depend on generating interest from industry and on securing approval for reimbursement from medical insurance companies. Industrial interest depends in large measure on the numbers of potential users. Expansion beyond the relatively small numbers of people who are locked-in, for example, to include individuals with high-level spinal cord injuries or severe cerebral palsy, could draw much greater commercial interest. Furthermore, as discussed again in Session 6 below, widespread application of BCI-based communication systems will depend also on cost, ease of training and use, and on careful attention to user satisfaction.

\section{SESSION 2: MATCHING THE BCI AND ITS INPUT TO THE USER}

Matching the user with his or her optimal BCI input features is essential if BCI's are ever to be broadly applied to the communication needs of users with different disabilities. Most BCI systems use EEG or single-unit features that originate mainly in somatosensory or motor areas of cortex. These areas may be severely damaged in people with stroke or neurogenerative disease. Use of features from other CNS regions may prove necessary. For EEG-based BCI's, comprehensive multielectrode recording, performed initially and then periodically, can reveal changes in the user's performance and/or the progression of disease, and can thereby guide selection of optimal recording locations and EEG features. Some brain areas may not prove to be useful: slow potential control is poor over parietal areas [22], and $\mu$ rhythms are largely limited to sensorimotor cortex. BCI systems should be flexible enough to use a variety of different EEG features as control signals. A system that can use slow potentials, $\mu$ or $\beta$ rhythms, P300 potentials, or single-unit activity alone or in combination is under development [52]. Such flexibility could provide a considerable practical advantage.

At present, only limited clinical data are available on BCI use by those with severe neuromuscular disabilities. The Thought Translation Device (TTD) [3], which uses slow cortical potentials (SCP's), and the Wadsworth BCI, which uses $\mu$ and $\beta$ rhythms [8], have been evaluated in small numbers of users with amyotrophic lateral sclerosis (ALS), stroke, spinal cord injury, cerebral palsy, or amputation. Controlled clinical trials in various user groups will be required to determine which BCI methods might be best for each group. These studies should compare the performances of different BCI systems and different electrophysiological inputs in comparable user groups. While a double-blind design is generally not practical in such work, comparable training procedures should be used and controls for placebo effects (e.g., [23]) should be incorporated. Because such studies are time consuming and costly, especially when they involve users with severe disabilities, they are likely to require the joint efforts of several centers.

The most obvious application of BCI technology in its present state of development is to locked-in patients. Totally locked-in patients should be differentiated from those who retain some minimal voluntary muscle functions, such as eye or eyelid movement or some facial muscle control. For the TTD, early training of ALS patients, before they lose all or most voluntary functions, has proved valuable because problems in learning SCP control from scratch can appear as the disease progresses. In addition, it may be necessary to change the feedback modality used by the BCI to accommodate the sensory capabilities of the user. Because the visual system may be compromised in certain user groups, BCI systems should be able to use other sensory modalities. Locked-in patients with poor control of eye movements might achieve better communication when tactile feedback is substituted for the standard visual feedback.

In addition to its use in locked-in states associated with brainstem strokes, ALS, or other degenerative diseases, BCI technology has been proposed as a possible communication system in autism, aphasia, and other severe communication disorders [24]. By bypassing compromised language areas in temperofrontal cortex, it could conceivably provide slow but less deviant or redundant communication. In patients who cannot master the alphabet, a pictorial system might prove an effective alternative.

With the exception of systems that depend on muscle control [2], [10], [25], and the possible exception of the P300 BCI [4], current BCI's depend on EEG or single-unit control that is acquired through operant conditioning procedures. Thus, thorough behavioral analysis of the learning phenomenon and environment is important. Such analysis requires extensive knowledge of learning theory, experience in its clinical application, and understanding of how a specific neurological impairment might influence learning. However, most current BCI's have been developed primarily by engineers and other technically oriented groups with limited expertise in behavioral principles and methods. High-level intellectual and cognitive functioning is probably not essential for successful BCI usage [26]. Nevertheless, issues such as optimal response selection, optimal reinforcement types and schedules, and optimal stimulus-response conditions, which are essential for successful shaping of any behavior (in this case, EEG or single-unit control) need much greater attention than they have received, and well-controlled clinical trials are essential. BCI development requires extensive interdisciplinary cooperation, between neuroscientists, engineers, psychologists, programmers, and rehabilitation specialists.

In addition to controlling the chosen electrophysiological features, users must simultaneously select the message to be communicated (e.g., specific letter or cursor movement direction) and observe the actual output from the system. This requires some division of attention, and might compromise control of the input features. Several studies indicate that such division is possible [27], [28]. Nevertheless, it may turn out that the capacity for automatization of brain responses such as slow cortical potentials or $\mu$ rhythms is limited. This problem could conceivably 
be overcome by switching to subcortical responses or by reducing the size of the cortical regions that produce the input used by the BCI system.

\section{SESSION 3: INVASIVE BCI METHODS}

This session reviewed the opportunities and difficulties associated with using invasive methods for obtaining BCI control signals. The starting point for this discussion was the observation that invasive methods are appropriate only if they are safe and if they provide significant improvement in function over noninvasive methods. The discussion focused on seven important questions.

First, what are the possible locations of implanted electrodes and what signals will they record? The motor cortex is an obvious choice for recording and should be considered in most cases because of its direct relevance to motor tasks, its relative accessibility compared to motor areas deeper in the brain, and the relative ease of recording from its large pyramidal cells. Other sites that might be considered include the supplementary motor cortex, subcortical motor areas, and the thalamus. Functional magnetic resonance imaging (fMRI), magnetoencephalography (MEG), and other functional imaging techniques could help identify appropriate areas for implantation. In addition, for some electrode types, neural recording during implantation can confirm that the sites selected are appropriate. The information in the recorded signals will depend on the location of the electrodes, and may be modified by sensory inputs to the recording area. Furthermore, the information will depend in as yet unknown ways on the functional use that is made of the signals, and this dependence may change with continued use. These factors all require further research. One issue of particular interest is how many cells need to be recorded simultaneously in order to get a meaningful signal. Estimates range from the expectation that one or two cortical neurons can provide useful information from an otherwise locked-in brain [29] to the belief that 50 to100 neurons will be needed to provide an information transfer rate that justifies an invasive procedure [18], [30]-[32]. These differing views lead to somewhat different research approaches.

Second, what are the options for obtaining stable recording capability over months and years? In small-brained animals such as rats and guinea pigs, stable single-unit recording has been maintained for long periods [30], [33]-[35]. In nonhuman primates stable recording has been maintained over months, and in selected instances over years [18], [36]-[38]. Recent results indicate that the cone electrode may provide stable recording in primates, including humans, for periods of years [36]. Other promising microelectrodes include microwires and micromachined microelectrode arrays [30], [33]-[35], [39], [40]. Further electrode development, combining the multisite capability of micromachined electrodes with the long-term stability of the cone electrode, is essential.

Third, which user groups might be best suited, by disability and/or need, for implanted electrodes? Patients who are locked in (e.g., by ALS) might benefit from invasive BCI technology if it is both safe and effective. Selected individuals with stroke, spinal cord injury, limb prostheses and other conditions might also benefit. Apart from the issues of safety and efficacy, the stigma sometimes associated with brain implants must be addressed and overcome. Individual preferences will play a significant role in decisions about implantable systems. To be justifiable, an implanted system must offer the individual a substantial functional advantage over conventional augmentative technologies and over noninvasive BCI methods. For example, a preliminary study is exploring noninvasive EEG-based operation of a neuroprosthesis that provides hand grasp [11].

Fourth, to what extent will the control provided by recorded neurons be able to be independent of the presence of normal feedback from other CNS areas? Implanted microelectrodes have been likened to a wiretap where the microelectrodes listen in to a normal conversation between cells. For users who are paralyzed or have other severe neuromuscular disabilities, it might be more appropriate to say that the implanted array of microelectrodes is a wiretap into a conversation in which one party has hung up. An effective BCI must provide feedback to the user and thereby substitute for the missing part of the conversation. The nervous system's ability to change so as to respond effectively to the new feedback provided by a BCI will have a major role in determining how well the communication system works. Studies indicate that the firing rates of individual neurons in motor cortex can be controlled using visual and auditory feedback [41]. Auditory prostheses research has shown that large populations of cells in the auditory system will modify their tuning specificity in response to electrical stimulation [42]. While the plasticity of the brain makes it difficult to predict the precise nature of the communication code, the expectation is that this plasticity will enhance communication efficiency.

Fifth, what other improvements in recording technology might help BCI development? Progress in several research areas is needed to make invasive methods a viable approach for a BCI. One area is signal acquisition, which includes recording methods, electrode design, artifact elimination, telemetry, and biomaterials. Other important research areas are the nature of information coding in the nervous system, the changes that occur with learning, and other aspects of integrative and cognitive neuroscience. Interdisciplinary cooperation is essential for this research. The research agenda includes further experimentation in primates, feasibility studies in humans, continued improvements in microelectrode design and telemetry, and studies of specific neuron populations and their ability to support BCI's with high information transfer rates.

Sixth, are other recently developed technologies such as $M E G$, fMRI, and positron emission tomography (PET) of possible use for BCI purposes? These technologies can have a significant role in patient evaluation and in preliminary identification of sites for implantation of invasive BCI recording electrodes. At the same time, given current understanding and equipment, it is not clear that these technologies could replace EEG or single-unit activity as the input for a portable and effective BCI. Nevertheless, in the future these and other imaging techniques, such as near-infrared photonic imaging, might support noninvasive BCI systems.

Seventh, what are the ethical issues that must be considered in implanting recording electrodes in human volunteers? Patients must be informed of the risks and potential benefits of any intervention, especially an invasive procedure with uncertain 
benefit to the individual and possibly serious risks. Volunteers with severe disabilities may tend to greatly overestimate the potential benefits, so that risks and uncertainties must be clearly and forcefully explained. On the other hand, many people may want to volunteer for research that provides no direct benefit to themselves beyond the knowledge that they are participating in a research project that might help others with similar conditions in the future. They should not be denied this opportunity. The Belmont Report [43] enunciates three basic ethical standards for the conduct of human research. The first, respect for persons, incorporates the idea that individuals are autonomous agents and should be free to make their own choice regarding participation after being given a full understanding of the risks and benefits. The second, beneficence, obligates the investigator to act in a way that will maximize benefit to the individual volunteer and/or the greater society while simultaneously minimizing the risk of harm. The third standard, justice, obligates the investigator to design studies so that the benefits and burdens of research are shared in a just way. An ethicist should be involved in the earliest phases of any human research developing or testing invasive BCI methods.

\section{SeSSiOn 4: Signal ANALYSiS}

The goal of signal analysis in a BCI system is to maximize the signal-to-noise ratio (SNR) of the EEG or single-unit features that carry the user's messages and commands. To achieve this goal, consideration of the major sources of noise is essential [44]. Noise has both nonneural sources (e.g., eye movements, EMG, $60-\mathrm{Hz}$ line noise) and neural sources (e.g., EEG features other than those used for communication). Noise detection and discrimination problems are greatest when the characteristics of the noise are similar in frequency, time or amplitude to those of the desired signal. For example, eye movements are of greater concern than EMG when a slow cortical potential is the BCI input feature because eye movements and slow potentials have overlapping frequency ranges. For the same reason, EMG is of greater concern than eye movements when a $\beta$ rhythm is the input feature. In the laboratory particularly, it is important to record enough information (e.g., topographical and spectral distributions) to permit discrimination between signal and noise. Non-neural noise such as EMG is of particular concern because a user's control over it can readily masquerade as actual EEG control. Non-neural noise produced by reflex activity may occur even in users who lack all voluntary muscle control. In this case, the nonneural noise will not support communication, but can degrade BCI performance by reducing the SNR. It is also important to distinguish between different neural features. The visual $\alpha$ rhythm is a source of noise when the $\mu$ rhythm is the feature being used for communication. While appropriate temporal and spatial filtering methods can help make such distinctions, signals from different sources might well have similar frequency spectra and similar spatial distributions (e.g., [45]). If nonneural and neural noise can be detected online, in the course of BCI operation, its impact on operation can be greatly reduced or eliminated. For example, in a BCI driven by slow cortical potentials, input contaminated by eye movements can be rejected [3]. This approach can also induce the user to reduce the production of such noise.
Numerous options are available for BCI signal processing. Ultimately, they need to be compared in on-line experiments that measure speed and accuracy. The new Graz BCI system [46], based on Matlab and Simulink, supports rapid prototyping of various methods. Different spatial filters and spectral analysis methods can be implemented in Matlab and compared in regard to their online performance.

Autoregressive (AR) model parameter estimation is a useful method for describing EEG activity, and can prove valuable for BCI applications (e.g., [7]-[9]). The AR model typically assumes a Gaussian process [15]. Because very small non-Gaussian residuals can markedly influence AR parameter estimation, these residuals should be assessed. When additive outlier contamination is present, a generalized robust maximum likelihood estimate (GM) can be valuable. This method is based on a modified Kalman filter. GM methods produce results similar to the typical AR estimate for Gaussian data, but perform better for non-Gaussian data. Bayesian algorithms, which can assess the certainty that the system's interpretation of the user's intention is correct, may also prove useful (e.g., [9]). They can arrest communication when this certainty falls below a criterion level, and thereby reduce errors in BCI performance.

Signal processing methods are important in BCI design, but they cannot solve every problem. While they can enhance the signal-to-noise ratio, they cannot directly address the impact of changes in the signal itself. Factors such as motivation, intention, frustration, fatigue, and learning affect the input features that the user provides. Thus, BCI development depends on appropriate management of the adaptive interactions between system and user, as well as on selection of appropriate signal processing methods.

\section{SESSION 5: BCI TRANSLATION AlgORITHMS}

A translation algorithm is a series of computations that transforms the BCI input features derived by the signal processing stage into actual device control commands. Stated in a different way, a translation algorithm takes abstract feature vectors that reflect specific aspects of the current state of the user's EEG or single-unit activity (i.e., aspects that encode the message that the user wants to communicate) and transforms those vectors into application-dependent device commands. Different BCI's use different translation algorithms (e.g., [3]-[9]). Each algorithm can be classified in terms of three key features: transfer function, adaptive capacity, and output. The transfer function can be linear (e.g., linear discriminant analysis, linear equations) or nonlinear (e.g., neural networks). The algorithm can be adaptive or nonadaptive. Adaptive algorithms can use simple handcrafted rules or more sophisticated machine-learning algorithms. The output of the algorithm may be discrete (e.g., letter selection) or continuous (e.g., cursor movement). The diversity in translation algorithms among research groups is due in part to diversity in their intended real-world applications. Nevertheless, in all cases the goal is to maximize performance and practicability for the chosen application.

Current consideration of alternative translation algorithms focuses primarily on those applicable to scalp-recorded EEG activity, because it is at present the only widely available BCI 
option for human users. As invasive technologies (e.g., the cone electrode [5] or intracortical or subdural arrays [16], [30]) evolve, extant algorithms will require additional evaluation and new algorithms will probably arise. EEG activity reflects the integrated activity of large populations of cortical neurons. If the input features extracted from this activity are to provide effective communication, they must have two or more discernible states that reflect the user's intentions and are accommodated to the domain and constraints of the application. The BCI might employ a single simple feature (e.g., amplitude in a specific frequency band at a specific scalp location), or a combination of multiple time and/or frequency domain features (e.g., slow cortical potentials, $\mu$ rhythms) produced by multiple physiological processes. The demands of the chosen application will help guide selection of a translation algorithm that provides an acceptable combination of speed and accuracy. Because the human brain is a highly adaptive controller that relies upon both predictive methods and feedback information, it is desirable and perhaps essential that BCI translation algorithms also be adaptive. One current algorithm adapts continually to the mean amplitude and/or variance of its EEG input features [44].

Whatever the nature and the computational power of a translation algorithm, it will not succeed without a comprehensive development and application strategy. Without such strategies, BCI development programs may degrade into optimizations of very abstract performance measures without real-world relevance. Thus, for example, the accuracy and speed of BCI-controlled curosr movement is meaningful only in the context of its performance in a specific target-selection protocol. While BCI research involves important theoretical issues and has important implications for the understanding of brain function, the primary impetus for it is the potential benefit to those with severe motor disabilities, and for this reason real-world relevance and success are crucial.

Objective methods for comparing different translation algorithms are essential for fostering further development and for synchronizing the multinational collaborative research programs that have arisen over the last several years. At present, such comparisons are often difficult, even for one specific application or within the same BCI system. This unsatisfactory situation could be improved by adoption of specific benchmark applications, uniform data sets, or standard procedures that will support comparisons between translation algorithms and between entire BCI systems, and will be accessible to all research groups (e.g., Fig. 1). Furthermore, because of the adaptive capacity of the brain and individual differences in this capacity, evaluation of translation algorithms should adopt appropriate statistical approaches (e.g., bootstrapping, cross-validation, forward prediction) and apply them in a sufficient number of users and in relevant applications. Particular algorithms may prove unsuitable for particular applications, and even the most sophisticated algorithms may fall short of expectations.

BCI development has begun to address real-world applications. Continued progress in development of these applications will require the combined effort of different laboratories and professions. Training protocols should be standardized within each laboratory and perhaps across laboratories. In addition, even the most sophisticated signal processing methods and translation algorithms are unlikely to be successful without involvement of neuroscientists, psychologists, physicians, and rehabilitative specialists who work with those who will be using BCI-based communication and control devices. Their guidance is essential to ensure that BCI technologies are not only theoretically effective but are also actually used by people for significant purposes in the real world.

\section{SESSION 6: APPLICATIONS OF BCI TECHNOLOGY}

As an essential prelude to addressing the potential practical uses of BCI technology, this session began by clarifying the distinction between BCI's and the applications to which they are applied. This is the difference between a tool, in this case a BCI, and its applications. A tool in the present context is a device that performs a specific function and can be applied to a wide variety of applications. A tool is specified by the manner in which it performs its function, and it is evaluated by the ease and effectiveness of its performance. The screwdriver, that most prototypical of tools, is designed to turn screws either clockwise or counterclockwise. This function remains the same, whatever the purpose served by the screws turned. In contrast, an application is a system that uses the tool to achieve some practical purpose. While an application may be described in terms of the tools it employs, its primary description focuses on the purpose it serves, and its evaluation focuses on how well it serves that purpose.

The BCI's described at this conference and in the papers in this issue are tools that record and analyze EEG or the activity of single cortical neurons. These tools can be used to move a cursor, select from among two or more possible choices, control a neuroprosthesis, etc. Discussions of the design and development of these tools inevitably focus on these possible applications and on the efficiency, reliability, and cost of specific tools in specific applications. Issues such as "How do we best move a cursor" or "How fast can we choose one of 26 characters?" are of primary concern. With satisfactory answers to these questions in hand, attention can turn to the real-life purposes the tools might serve.

Present BCI's can be classified into two groups according to the nature of the signals they use as input. Some depend on user control of endogenous electrophysiological activity, such as amplitude in a specific frequency band in EEG recorded over a specific cortical area (e.g., $\mu$ or $\beta$ rhythms recorded over sensorimotor cortex [6]-[9]). Others depend on user control of exogenous electrophysiological activity, that evoked by specific stimuli (e.g., amplitude of the P300 potential produced in response to letter flash [4]). Endogenous BCI's provide a better fit to a control model because the trained user exercises direct control over the environment. On the other hand, these BCI's often require extensive training. Exogenous BCI's may not require extensive training, but do require a somewhat structured environment (e.g., stereotyped visual input). For example, an endogenous BCI may enable a user to move a cursor to any point in a two-dimensional space, while an exogenous BCI may constrain a user to the choices presented by a display.

BCI tools have potential applications spanning at least five different areas: verbal communication, activities of daily living, environmental control, locomotion, and exercise. In choosing 
among these areas, the needs and priorities of the anticipated user should be the primary concern. Developers must guard against the tendency to approach the parameters of the tools and their applications as an abstract design exercise. A BCI and its applications should be optimized for each individual user or user group. At the same time, the optimization process should be as objective and standardized as possible. For each user, the BCI and its application(s) should be embedded in a behavioral program with well-defined objectives. A thorough behavioral analysis that addresses the needs, desires, and primary motivators of the user and/or her or his caregivers is essential. BCI development should incorporate not simply technical and electrophysiological principles, but well-defined learning principles as well.

One approach to application development would begin with a matrix that lists the tools (i.e., the different BCI's) on one axis and their characteristics (e.g., speed, accuracy, training needed, demand on attention, etc.) on the other axis. A second matrix would list applications on one axis and their requirements (e.g., speed and accuracy needed, attention required, etc.) on the other. The rows and columns of these two matrices could be combined to produce a BCI-to-application mapping matrix that would aid developers in designing applications and providing them to individual users. This matrix might also aid clinicians in exploring collaborative development, or integration, of several different BCI's to better serve particular applications [47]. In addition, users with a progressive disorder such as ALS might be provided with a BCI that matches the characteristics of an existing conventional augmentative communication interface. Then, as disability progresses and the conventional interface loses its usefulness, the user might make a smooth transition to the BCI.

The recent development and commercial application of the Freehand Functional Electrical Stimulation system (FES) at Case Western Reserve University, Cleveland, $\mathrm{OH}$, illustrates the conditions necessary for success (e.g., [48]). These conditions include: stabilization of all aspects of design and documentation, a well-defined user population, standardized training protocols, demonstration of the feasibility of the specific application, well-defined outcome measures that document successful usage and performance, regulatory assessment, multicenter assessment, and identification of manufacturing partners and commercialization strategy. Satisfying these conditions requires collaborative interactions with the users, who must know how to use the technology and be persuaded that the technology is both useful and safe. Also essential is the cooperation of the relevant health care professionals, who must be persuaded that the risk/benefit ratio is favorable, that the technology is safe and useful, and that it is equal or superior to available alternatives. Finally, whoever is paying the bill (usually an insurance company) must be convinced that the long-term savings will offset both the initial costs and the operating costs of the system.

Among the factors that impede development of BCI applications are: that the essential neuroscientific and psychological foundations of the field are not sufficiently developed, that current EEG recording methods are somewhat cumbersome and susceptible to noise from various sources, that current BCI's have limited resolution (e.g., binary selection is a weak substitute for continuous or multilevel selection), that close interdisciplinary collaboration (i.e., engineering, neuroscience, psychology, computer science, rehabilitation) is still rare, and that access to appropriate users with substantial interest in obtaining improved function is as yet inadequate. Access to a sufficient number of appropriate and motivated users is a particular challenge.

The panel did not recommend selection of a single prototype application for all research projects. Although a prototype application would allow objective comparison between BCI systems, the choice of application would place an arbitrarily high priority on specific performance characteristics while downplaying others that might be of equal or greater importance for other applications. A better alternative would be a standard set of benchmark applications that would together quantify the different performance characteristics of each BCI, including accuracy, speed of operation, etc. The results of such a standard and comprehensive evaluation would help in matching BCI to application to individual user.

\section{SUPPORT FOR BCI RESEARCH AND DEVELOPMENT}

Further development of BCI technology will depend on both basic and applied research. Basic research efforts that elucidate the mechanisms underlying and controlling EEG rhythms, cortical single-unit activity, and other electrophysiological phenomena, that develop processing methods that improve SNR, or that provide other insights into the physiological, psychological processes and engineering principles involved in BCI operation will be an essential element in future progress. Public and private entities that support basic biomedical and engineering research are likely sources of support for this work. Of particular interest in this context is the new initiative on bioengineering research at the National Institutes of Health (NIH) [49] (e.g., [50]). This program emphasizes interdisciplinary research and is thus particularly well-suited for BCI research efforts.

Applied research-the development and evaluation of particular BCI's in particular applications-is also essential. At present, the primary impetus for this work is the need of those with severe neuromuscular disabilities, those who lack the voluntary muscle control needed to use conventional augmentative communication systems. While their need is great, their numbers are small. As a result, BCI development is as yet of limited commercial interest and depends for support mainly on public and private nonprofit entities. Because these institutions traditionally focus on basic research, support for applied BCI research has been difficult to obtain. In recent years, the National Center for Medical Rehabilitation Research of the NIH has recognized this problem and has begun to provide support for research programs that are primarily applied rather than basic. The NIH Small Business Innovation Research Grant Program [51] also provides support for applied research that has as yet only limited commercial potential. The National Institute of Disability and Rehabilitation Research is another potential source of support. Other NIH grant mechanisms and the specific interests of the different institutes at NIH are described on NIH's home page (www.nih.gov). In Europe, the European Union and national agencies (e.g., the Deutsche Forschungsgemeinschaft (DFG) in Germany) have also begun to provide support for such applied research. In the future, if 
and when the speed and accuracy of BCI technologies increase enough to make them useful for larger populations with less severe disabilities, private industry is likely to display greater interest and to provide substantial support.

\section{CONCLUSION}

A brain-computer interface is a communication and control channel that does not depend on the brain's normal output pathways of peripheral nerves and muscles. At present, the main impetus to BCI research and development is the expectation that BCI technology will be valuable for those whose severe neuromuscular disabilities prevent them from using conventional augmentative communication methods. These individuals include many with advanced amyotrophic lateral sclerosis (ALS), brainstem stroke, and severe cerebral palsy.

Current BCI's record electrophysiological signals using noninvasive or invasive methods. Noninvasive BCI's use scalp-recorded EEG rhythms or evoked potentials, while invasive BCI's use single-unit activity recorded within cortex or EEG recorded subdurally. They have maximum information transfer rates of 5-25 b/min and are being used to control cursor movement or to select letters or icons.

Like other communication and control systems, BCI's have inputs, outputs, and translation algorithms that convert the former to the latter. BCI operation depends on the interaction of two adaptive controllers, the user's brain, which produces the input (i.e., the electrophysiological activity measured by the BCI system) and the system itself, which translates that activity into output (i.e., specific commands that act on the external world). Successful BCI operation requires that the user acquire and maintain a new skill, a skill that consists not of muscle control but rather of control of EEG or single-unit activity.

BCI inputs include slow cortical potentials, P300 evoked potentials, $\mu$ and $\beta$ rhythms from sensorimotor cortex, and single unit activity from motor cortex. Recording methodologies seek to maximize signal-to-noise ratio. Noise consists of EMG, EOG, and other activity from sources outside the brain, as well as brain activity different from the specific rhythms or evoked potentials that comprise the BCI input. A variety of temporal and spatial filters can reduce such noise and thereby increase the signal-to-noise ratio. BCI translation algorithms include linear equations, neural networks, and numerous other classification techniques. The most difficult aspect of their design and implementation is the need for continuing adaptation to the characteristics of the input provided by the user.

BCI development depends on close interdisciplinary cooperation between neuroscientists, engineers, psychologists, computer scientists, and rehabilitation specialists. It would benefit from general acceptance and application of objective methods for evaluating translation algorithms, user training protocols, and other key aspects of BCI operations. Evaluations in terms of information transfer rate and in terms of usefulness in specific applications are both important. Appropriate user populations must be identified, and BCI applications must be configured to meet their most important needs. The assessment of needs should focus on the actual desires of individual users rather than on preconceived notions about what these users ought to want.
Similarly, evaluation of specific applications ultimately rests on the extent to which people actually use them in their daily lives.

Continuation and acceleration of recent progress in BCI research and development requires increased focus on the production of peer-reviewed research articles in high quality journals. Research would also benefit from identification and widespread utilization of appropriate venues for presentations (e.g., the Society for Neuroscience Annual Meeting), and from appropriately conservative response to media attention. For the near future, research funding will depend primarily on public agencies and private foundations that fund research directed at the needs of those with severe motor disabilities. With further increases in speed, accuracy, and range of applications, BCI technology could become applicable to larger populations and could thereby engage the interest and resources of private industry.

\section{ACKNOWLEDGMENT}

The authors thank the National Center for Medical Rehabilitation Research, NICHD, NIH, which sponsored the workshop, the Eastern Paralyzed Veterans Association and the Whitaker Foundation which provided additional support, the Wadsworth Center of the New York State Department of Health which organized the workshop, and the Rensselaerville Institute near Albany, NY, which provided and maintained an ideal environment.

The panels that planned and led the six discussion sessions were comprised of the following people:

Session 1: J. R. Wolpaw (Chair), E. Curran, A. Gevins, A. M. Junker, S. G. Mason, C. J. Robinson.

Session 2: N. Birbaumer (Chair), F. Cincotti, I. Goncharova, S. Makeig, C. Neuper, F. Pauri, J. A. Pineda.

Session 3: W. J. Heetderks (Chair), L. Bianchi, J. K. Chapin, G. Gaal, R. Isaacs, P. R. Kennedy, S. P. Levine. Session 4: D. J. McFarland (Chair), J. H. Bayliss, G. E. Birch, C. Guger, T. Hinterberger, W. D. Penny.

Session 5: P. H. Peckham (Chair), G. Schalk (Discussion Leader), J. H. Bayliss (Recorder), C. Anderson, G. Fabiani, A. Kostov, J. Perelmouter.

Session 6: E. Donchin (Chair), B. Z. Allison, J. E. Huggins, A. Kuebler, R. T. Lauer, M. S. Middendorf, M. Polak.

\section{REFERENCES}

[1] H. Berger, "Uber das Electrenkephalogramm des Menchen," Arch Psychiat Nervenkr, vol. 87, pp. 527-570, 1929.

[2] J. J. Vidal, "Real-time detection of brain events in EEG," Proc. IEEE, vol. 65, pp. 633-664, May 1977.

[3] N. Birbaumer et al., "The thought translation device (TTD) for completely paralyzed patients," IEEE Trans. Rehab. Eng., vol. 8, pp. 190-193, June 2000.

[4] E. Donchin et al., "The mental prosthesis: Assessing the speed of a P300based brain-computer interface," IEEE Trans. Rehab. Eng., vol. 8, pp. 174-179, June 2000.

[5] P. R. Kennedy et al., "Direct control of a computer from the human central nervous system," IEEE Trans. Rehab. Eng., vol. 8, pp. 198-202, June 2000.

[6] A. Kostov and M. Polak, "Parallel man-machine training in development of EEG-based cursor control," IEEE Trans. Rehab. Eng., vol. 8, pp. 203-205, June 2000.

[7] G. Pfurtscheller et al., "Current trends in Graz brain-computer interface (BCI) research," IEEE Trans. Rehab. Eng., vol. 8, pp. 216-219, June 2000 . 
[8] J. R. Wolpaw et al., "Brain-computer interface research at the Wadsworth Center," IEEE Trans. Rehab. Eng., vol. 8, pp. 222-226, June 2000.

[9] W. D. Penny et al., "EEG-based communication: A pattern recognition approach," IEEE Trans. Rehab. Eng., vol. 8, pp. 214-215, June 2000.

[10] M. Middendorf et al., "Brain-computer interfaces based on steady-state visual-evoked response," IEEE Trans. Rehab. Eng., vol. 8, pp. 211-214, June 2000.

[11] R. T. Lauer et al., "Applications of cortical signals to neuroprosthetic control: A critical review," IEEE Trans. Rehab. Eng., vol. 8, pp. 205-208, June 2000.

[12] J. A. Pineda et al., "The effects of self-movement, observation, and imagination on $\mu$ rhythms and readiness potentials (RP's): Toward a brain-computer interface (BCI)," IEEE Trans. Rehab. Eng., vol. 8, pp. 219-222, June 2000.

[13] F. Babilioni et al., "Linear classification of low-resolution EEG patterns produced by imagined hand movements," IEEE Trans. Rehab. Eng., vol 8, pp. 186-188, June 2000

[14] S. Makeig et al., "A natural basis for efficient brain-actuated control," IEEE Trans. Rehab. Eng., vol. 8, pp. 208-211, June 2000.

[15] G. E. Birch and S. G. Mason, "Brain-computer interface research at the Neil Squire Foundation," IEEE Trans. Rehab. Eng., vol. 8, pp. 193-195, June 2000.

[16] S. P. Levine et al., "A direct brain interface based on event-related potentials," IEEE Trans. Rehab. Eng., vol. 8, pp. 180-185, June 2000.

[17] J. D. Bayliss and D. H. Ballard, "A virtual reality testbed for brain-computer interface research," IEEE Trans. Rehab. Eng., vol. 8, pp. 188-190, June 2000.

[18] R. E. Isaacs et al., "Work toward real-time control of a cortical neura prosthesis," IEEE Trans. Rehab. Eng., vol. 8, pp. 196-198, June 2000.

[19] L. E. Roberts et al., "Self-report during feedback regulation of slow cortical potentials," Psychophysiol, vol. 26, pp. 392-403, 1989.

[20] C. E. Shannon and W. Weaver, The Mathematical Theory of Communication. Urbana, IL: Univ. Illinois Press, 1964.

[21] J. R. Pierce, An Introduction to Information Theory. New York: Dover vol. 1080, pp. 145-165.

[22] B. Rochstroh et al., Slow Brain Potentials and Behavior, 2nd ed. Baltimore, MD: Urban and Schwarzenberg, 1989.

[23] A. Harrington, The Placebo Effect. Cambridge, MA: Harvard Univ Press, 1997.

[24] N. Birbaumer, "Rain Man's revelations," Nature, vol. 399, pp. 211-212, 1999.

[25] E. E. Sutter, "The brain response interface: Communication through visually-induced electrical brain responses," J. Microcomput. Appl., vol. 15, pp. 31-45, 1992.

[26] S. Holzapfel et al., "Behavioral psychophysiological intervention in a mentally retarded epileptic patient with brain lesion," Appl. Psychophysiol. Feedback, vol. 23, no. 3, pp. 189-202, 1998.

[27] J. Perelmouter et al., "Language support program for thought-translation-devices," Automedica, vol. 18, pp. 67-84, 1999.

[28] L. A. Miner et al., "Answering questions with an electroencephalogrambased brain-computer interface," Arch. Phys. Med. Rehab., vol. 79, pp. 1029-1022, 1998

[29] P. R. Kennedy and R. A. Bakay, "Restoration of neural output from a paralyzed patient by a direct brain connection," Neuroreport, vol. 9, pp. $1707-1711,1998$

[30] J. K. Chapin et al., "Real-time control of a robot arm using simultaneously recorded neurons in the motor cortex," Nat. Neurosci., vol. 2, pp. 664-760, 1999

[31] J. A. Hoffer et al., "Neural signals for command control and feedback in functional neuromuscular stimulation: A review," J. Rehab. Res. Dev., vol. 33, pp. 145-157, 1996.

[32] S. Lin et al., "Self-organization of firing activities in monkey's motor cortex: Trajectory computation from spike signals," Neural Comput., vol. 9, pp. 607-621, 1997.

[33] D. J. Woodward et al., "Mesolimbic neuronal activity across behavioral states," Ann. New York Acad. Sci., vol. 877, pp. 91-112, 1999.

[34] J. C. Williams et al., "Long-term neural recording characteristics of wire microelectrode arrays implanted in cerebral cortex," Brain Res. Brain Res. Protoc., vol. 4, pp. 303-313, 1999.

[35] X. Liu et al., "Stability of the interface between neural tissue and chronically implanted intracortical microelectrodes," IEEE Trans. Rehab. Eng., vol. 7, pp. 315-326, 1999.

[36] P. R. Kennedy and R. A. Bakay, "Activity of single action potentials in monkey motor cortex during long-term task learning," Brain Res., pp. 251-254, 1997.
[37] E. M. Schmidt et al., "Long-term implants of Parylene-C coated microelectrodes," Med. Biol. Eng. Comput., vol. 26, pp. 96-101, 1988.

[38] E. M. Maynard et al., "Neuronal interactions improve cortical population coding of movement direction," J. Neurosci., vol. 19, pp 8083-8093, 1999

[39] P. J. Rousche and R. A. Normann, "Chronic recording capability of the Utah intracortical electrode array in cat sensory cortex," J. Neurosci. Math., vol. 82, pp. 1-15, 1998.

[40] A. C. Hoogerwerf and K. D. Wise, "A three-dimensional micorelectrode array for chronic neural recording," IEEE Trans. Biomed. Eng., vol. 41, pp. 1136-1146, 1994.

[41] E. M. Schmidt, "Single neuron recording from motor cortex as a possible source of signals for control of external devives," Ann. Biomed. Eng., vol. 8, pp. 339-349, 1980.

[42] M. Vollmer et al., "Temporal properties of chronic cochlear electrical stimulation determine temporal resolution of neurons in cat inferior colliculus," J. Neurophysiol., vol. 82, pp. 2883-2909, 1999.

[43] _ Ethical principles and guidelines for the protection of human subjects of research, report of the national commission for the protection of human subjects and biomedical and behavioral research, Apr. 18 , 1979, Available: http://grants.nih.gov/grants/oprr/humansubjects/guidance/belmont.htm [Online].

[44] D. J. McFarland et al., "Design and operation of an EEG-based braincomputer interface with digital signal processing technology," Behav Res. Meth. Inst. Comp., vol. 29, pp. 337-345, 1997.

[45] G. Floran et al., "Do changes in coherence always reflect changes in functional coupling?," Electroenceph. Clin. Neurophysiol., vol. 106, pp. 87-91, 1998.

[46] A. Guger et al., "Design of an EEG-based brain-computer interface (BCI) from standard components running in real-time under Windows," Biomed. Tech., vol. 44, pp. 12-16, 1999.

[47] G. E. Jacques et al., "Application of quality function deployment in rehabilitation engineering," IEEE Trans. Rehab. Eng., vol. 2, pp. 158-164, Sept. 1994.

[48] K. L. Kilgore et al., "An implanted upper-extremity neuroprothesis: Follow-up of five patients," J. Bone Joint Surg., vol. 79A, pp. 533-541, 1997.

[49] - Biomedical Engineering (BECON), NIH Office of Extramural Research, Available: (http://grants.nih.gov/grants/becon/becon/htm [Online].

[50] - Bioengineering Research Grants, Available: http://grants.nih.gov.grants//guide.pa-files/PAR-99-009.html [Online].

[51] Small Business Funding Opportunities, NIH Office of Extramural Research. Available: http://www.nih.gov.grants/funding/sbir/htm [Online]

[52] J. R. Wolpaw et al., "BCI2000: A general purpose brain-computer interface (BCI)," Soc. Neurosci. Abstr., 2000, in press.

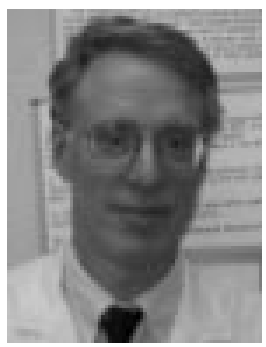

Jonathan R. Wolpaw received the A.B. degree from Amherst College, MA, in 1966 and the M.D. degree from Case Western Reserve University, Cleveland, $\mathrm{OH}$, in 1970 . He completed a residency in neurology at the University of Vermont, Burlington, and fellowship training in neurophysiology research at the $\mathrm{Na}-$ tional Institutes of Health.

He is Chief of the Laboratory of Nervous System Disorders and a Professor at the Wadsworth Center of the New York State Department of Health and the State University of New York. His primary research interest are use of operant conditioning of spinal reflexes as a new model for defining the plasticity underlying a simple form of learning in vertebrates and development of EEG-based communication technology for those with severe motor disabilities.

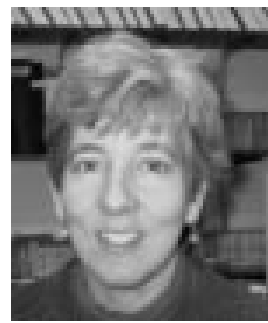

Theresa M. Vaughan received the B.A. degree from the State University of New York at Binghamton in 1976.

She works at the Wadsworth Center of the New York State Department of Health. She has been involved in the Wadsworth Brain-Computer Interface Project since 1990 and has been the Project Coordinator since 1991 